\title{
The influence of mechanical vibration moulding process on thermal conductivity and diffusivity of Al-TiC particulate reinforced composites
}

\begin{abstract}
Titanium carbide particulate reinforced composites were fabricated by vibrational moulding sand casting technique. Titanium carbide particulate added as a reinforcement phase in the LM6 alloy matrix and the percentage of addition varied from $0.2,0.6,1$ and $2 \%$ on weight fraction basis. The thermal conductivity and diffusivity of $\mathrm{TiC}$ composites were determined. Increasing frequency of vibration and $\mathrm{TiC}$ content helps to improve density, thermal conductivity, thermal diffusivity and purification of the grain boundaries. The dispersed particles increased the thermal conductivity and diffusivity according to an effective media theory.
\end{abstract}

Keyword: Composite materials; Conductivity; Diffusivity 